01;09

\title{
Расчет импедансных вибраторных антенн
}

\author{
() С.И. Эминов
}

Новгородский государственный университет им. Ярослава Мудрого, Великий Новгород

E-mail: eminovsi@mail.ru

Поступило в Редакцию 27 декабря 2016 г.

Метод аналитического обращения гиперсингулярного уравнения применен к уравнению импедансной вибраторной антенны. Разработан численный метод решения уравнения. Продемонстрирована эффективность метода.

DOI: 10.21883/PJTF.2017.13.44805.16638

Задачей данной работы является сведение интегрального уравнения импедансной вибраторной антенны к уравнению Фредгольма второго рода и построение эффективного численного метода на основе полиномов Чебышева второго рода.

На поверхности вибраторной антенны выполняется граничное условие вида [1]

$$
E_{\tau}\left(j_{\tau}\right)+E_{\tau}^{0}=Z j_{\tau}
$$

где $E_{\tau}\left(j_{\tau}\right)$ - вторичное электрическое поле, создаваемое поверхностными токами $j_{\tau}, E_{\tau}^{0}-$ первичное электрическое поле, $Z-$ поверхностный импеданс. Если поверхность антенны идеально-проводящая, то правая часть уравнения (1) равна нулю. Этот случай рассмотрен в работе [2]. Наличие правой части - оператора умножения на постоянную, существенно меняет структуру уравнения, усложняя ее. Поскольку единичный оператор ограничен в пространстве $L_{2}[-1,1]$, он не является вполне непрерывным. Преобразуя левую часть, уравнение (1) можно 
записать как гиперсингулярное интегро-дифференциальное уравнение вида [2]

$$
\begin{aligned}
(A u)(\tau) & +\alpha u(\tau)+\beta(L u)(\tau)+(K u)(\tau) \\
& \equiv \frac{1}{\pi} \frac{\partial}{\partial \tau} \int_{-1}^{1} u(t) \frac{\partial}{\partial t} \ln \frac{1}{|\tau-t|} d t \\
& +\alpha u(\tau)+\beta \int_{-1}^{1} u(t) \ln \frac{1}{|\tau-t|} d t+\int_{-1}^{1} K(\tau, t) u(t) d t=f(\tau), \\
& -1 \leqslant \tau \leqslant 1,
\end{aligned}
$$

где $\alpha$ и $\beta-$ постоянные величины, ядро $K(\tau, t)$ является непрерывной функцией. Если поверхность антенны является идеально проводящей, то $\alpha$ равно нулю. Для импедансных вибраторных антенн $\alpha$ отлична от нуля.

Теория и методы решения уравнения (2) основаны на свойствах гиперсингулярного интегро-дифференциального оператора

$$
(A u)(\tau)=\frac{1}{\pi} \frac{\partial}{\partial \tau} \int_{-1}^{1} u(t) \frac{\partial}{\partial t} \ln \frac{1}{|\tau-t|} d t .
$$

Аналитическое обращение этого гиперсингулярного оператора получено в работе [2], а свойства изучены в работе [3]. Из этой работы приведем один результат, который нам потребуется.

Оператор $A$ является симметричным положительно-определенным оператором в гильбертовом пространстве $L_{2}[-1,1]$ и имеет плотную область определения. Обратный к нему оператор определяется формулой

$$
\left(A^{-1} f\right)(\tau)=\frac{1}{\pi} \int_{-1}^{1} f(t) \ln \left|\frac{\tau-t}{1-\tau t+\sqrt{1-\tau^{2}} \sqrt{1-\tau^{2}}}\right| d t
$$

и является не только ограниченным, но и вполне непрерывным оператором в пространстве $L_{2}[-1,1]$. Для любого ограниченного в пространстве $L_{2}[-1,1]$ оператора $B$ оператор $T=A^{-1} B$ вполне непрерывен в энергетическом пространстве $H_{A}$ симметричного положительноопределенного оператора $A$. 
Умножая обе части уравнения (2) на оператор $A^{-1}$, получим

$$
u(\tau)+\alpha\left(A^{-1} u\right)(\tau)+\beta\left(A^{-1} L u\right)(\tau)+\left(A^{-1} K u\right)(\tau)=A^{-1} f .
$$

Одно из важных свойств этой теоремы заключается в том, что уравнение (5) является уравнением Фредгольма второго рода.

Введем систему функций

$$
\varphi_{n}(\tau)=\sqrt{\frac{2}{\pi n}} \sin [n \arccos (\tau)]=\sqrt{\frac{2}{\pi n}} \sqrt{1-\tau^{2}} U_{n}(\tau), n=1,2,3, \ldots,
$$

$U(\tau)$ - полиномы Чебышева второго рода: $U_{1}(\tau)=1, U_{2}(\tau)=2 \tau$, $U_{3}(\tau)=4 \tau^{2}-1$ и т.д. Эта система функций обладает тремя замечательными свойствами. Она является полной и ортонормированной в следующем смысле:

$$
\left(A \varphi_{n}, \varphi_{m}\right)=\left\{\begin{array}{l}
1, m=n \\
0, m \neq n
\end{array},\right.
$$

здесь $(\cdot, \cdot)$ означает скалярное произведение в $L_{2}[-1,1]$ :

$$
(f(\tau), g(\tau))=\int_{-1}^{1} f(\tau) \overline{g(\tau)} d \tau .
$$

Наконец, каждая функция $\varphi_{n}(\tau)$ удовлетворяет условию Мейкснера на ребре. Благодаря последнему свойству система функций (6) широко применяется как в задачах расчета антенн, так и в теории дифракции.

Сформулируем метод Галеркина. Приближенное решение уравнения (2) ищется в виде

$$
u(\tau)=\sum_{n=1}^{N} c_{n} \varphi_{n}(\tau)
$$

Подставим (8) в (2) и умножим скалярно в пространстве $L_{2}[-1,1]$ на базисные функции $\varphi_{1}, \varphi_{2}, \ldots, \varphi_{N}$. В результате получим систему линейных алгебраических уравнений

$$
c_{n}+\alpha \sum_{m=1}^{N} c_{m} I_{m n}+\beta \sum_{m=1}^{N} c_{m} L_{m n}+\sum_{m=1}^{N} c_{m} K_{m n}=f_{n}, n=1,2, \ldots, N,
$$

Письма в ЖТФ, 2017, том 43, вып. 13 
где

$I_{m n}=\left(\varphi_{m}, \varphi_{n}\right), \quad L_{m n}=\left(L \varphi_{m}, \varphi_{n}\right), \quad K_{m n}=\left(K \varphi_{m}, \varphi_{n}\right), \quad f_{n}=\left(f, \varphi_{n}\right)$.

После решения системы (9) по формуле (8) найдем приближенное решение уравнения (5) или (2). Из сформулированной теоремы следует, что приближенное решение будет сходиться к точному решению в пространстве $H_{A}$ и в $L_{2}[-1,1]$.

Рассмотрим вопрос вычисления матричных элементов. Матрицы единичного оператора и интегрального оператора $L$ с логарифмической особенностью в ядре удается найти в аналитическом виде. Вначале найдем преобразование Фурье базисных функций, как табличные интегралы [4]

$$
\begin{gathered}
\tilde{\varphi}_{2 i-1}(x)=\int_{-1}^{1} \varphi_{2 i-1}(t) \exp (i t x) d t=(-1)^{i-1} \sqrt{2 \pi(2 i-1)} \frac{J_{2 i-1}(x)}{x}, \\
\tilde{\varphi}_{2 i}(x)=\int_{-1}^{1} \varphi_{2 i}(t) \exp (i t x) d t=i(-1)^{i-1} \sqrt{4 \pi i} \frac{J_{2 i}(x)}{x}
\end{gathered}
$$

где $J_{2 i-1}-$ функция Бесселя. Применим формулу Парсеваля и сведем вычисление матричных элементов к вычислению интеграла по бесконечному промежутку

$$
\left(\varphi_{2 i-1}, \varphi_{2 j-1}\right)=(-1)^{i+j} 2 \sqrt{(2 i-1)(2 j-1)} \int_{0}^{+\infty} \frac{1}{x^{2}} J_{2 i-1}(x) J_{2 j-1}(x) d x .
$$

Последний интеграл также находится аналитически, как табличный интеграл [4]

$$
\begin{aligned}
\left(\varphi_{2 i-1}, \varphi_{2 j-1}\right) & =(-1)^{i+j} \frac{1}{2} \sqrt{(2 i-1)(2 j-1)} \\
& \times \frac{\Gamma(i+j-3 / 2)}{\Gamma(i-j+3 / 2) \Gamma(j-i+3 / 2) \Gamma(i+j+1 / 2)} .
\end{aligned}
$$

С учетом следующих двух свойств гамма-функции

$$
\Gamma(x+1)=x \Gamma(x), \Gamma\left(x+\frac{1}{2}\right) \Gamma\left(-x+\frac{1}{2}\right)=\frac{\pi}{\cos (\pi x)}
$$

Письма в ЖТФ, 2017, том 43, вып. 13 
представление (11) приводится к виду

$$
\left(\varphi_{2 i-1}, \varphi_{2 j-1}\right)=\frac{\sqrt{(2 i-1)(2 j-1)}}{2 \pi(i-j+1 / 2)(j-i+1 / 2)(i+j-3 / 2)(i+j-1 / 2)} .
$$

Для нахождения матричных элементов оператора $L$ применяется тождество [4]

$$
\begin{aligned}
& \ln \frac{1}{|\tau-t|}=C+\int_{0}^{1} \frac{\cos [x(\tau-1)]-1}{x} d x+\int_{1}^{+\infty} \frac{\cos [x(\tau-t)]}{x} d x, \\
& C=0.5772 .
\end{aligned}
$$

Используя преобразование Фурье базисных функций и применяя табличный интеграл [4]

$$
\begin{aligned}
& \int_{0}^{+\infty} x^{\alpha-1} J_{m}(c x) J_{n}(c x) d x=2^{\alpha-1} c^{-\alpha} \\
& \times \frac{\Gamma(1-\alpha) \Gamma((m+n+\alpha) / 2)}{\Gamma(1+(n-m-\alpha) / 2) \Gamma(1+(m-n-\alpha) / 2) \Gamma(1+(m+n-\alpha) / 2)},
\end{aligned}
$$

найдем матричные элементы интегрального оператора $L$ с логарифмической особенностью в ядре

$$
\begin{gathered}
\left(L \varphi_{2 i-1}, \varphi_{2 j-1}\right)=\frac{1}{2} \ln 2+\frac{1}{8}, \text { если } i=j=1, \\
\left(L \varphi_{2 i-1}, \varphi_{2 j-1}\right)=\frac{1}{8 i(i-1)}, \text { если } i=j>1 \\
\left(L \varphi_{2 i-1}, \varphi_{2 j-1}\right)=-\frac{\sqrt{(2 i-1)(2 j-1)}}{4(i+j)(i+j-1)(i+j-2)}, \text { если } j=i-1, \\
\left(L \varphi_{2 i-1}, \varphi_{2 j-1}\right)=\left(\varphi_{2 i-1}, L \varphi_{2 j-1}\right), \\
\left(L \varphi_{2 i-1}, \varphi_{2 j-1}\right)=0, \text { если }|i-j|>1
\end{gathered}
$$

Письма в ЖТФ, 2017, том 43, вып. 13 
Зависимость приближенного решения от числа базисных функций

\begin{tabular}{r|c|r|c}
\hline$N$ & $\alpha=0, \beta=1 / \pi$ & $N$ & $\alpha=1 / \pi, \beta=1 / \pi$ \\
\hline 1 & 0.3339772 & 1 & 0.2154801 \\
2 & 0.3275391 & 2 & 0.2089073 \\
3 & 0.3276998 & 3 & 0.2025135 \\
4 & 0.3276989 & 4 & 0.2021649 \\
5 & 0.3276989 & 5 & 0.2022841 \\
10 & 0.3276989 & 10 & 0.2022471 \\
20 & 0.3276989 & 20 & 0.2022492 \\
40 & 0.3276989 & 40 & 0.2022493 \\
80 & 0.3276989 & 80 & 0.2022493
\end{tabular}

Формулы (15)-(19) в таком полном виде приводятся впервые в научной литературе. Наконец, матрица интегрального оператора $K$ с непрерывным ядром эффективно считается на ЭВМ.

Эффективность метода продемонстрируем на следующем модельном уравнении:

$$
\begin{gathered}
(A u)(\tau)+\alpha u(\tau)+\beta(L u)(\tau) \equiv \frac{1}{\pi} \frac{\partial}{\partial \tau} \int_{-1}^{1} u(t) \frac{\partial}{\partial t} \ln \frac{1}{|\tau-t|} d t+ \\
+\alpha u(\tau)+\beta \int_{-1}^{1} u(t) \ln \frac{1}{|\tau-t|} d t=0.5, \quad-1 \leqslant \tau \leqslant 1 .
\end{gathered}
$$

В таблице приводятся значения решения интегрального уравнения в нуле, т.е. $u(0)$. Таблица характеризует быструю сходимость метода Галеркина. Это связано с двумя важными причинами: во-первых, все матричные элементы найдены аналитически и, во-вторых, матрица интегрального оператора $L$ является трехдиагональной.

Таким образом, доказано, что интегральное уравнение эквивалентно уравнению Фредгольма второго рода. Решена задача вычисления матричных элементов и разработан численный метод решения интегрального уравнения. На модельном примере показана высокая эффективность метода.

Письма в ЖТФ, 2017, том 43, вып. 13 


\section{Список литературы}

[1] Васильев Е.Н. Возбуждение тел вращения. М.: Радио и связь, 1987.

[2] Эминов С.И. // Письма в ЖТФ. 2004. Т. 30. В. 22. С. 8-16.

[3] Эминова В.С., Эминов С.И. // Журнал вычислительной математики и математической физики. 2016. Т. 56. № 3. С. 432-440.

[4] Прудников А.П., Брычков Ю.А., Маричев О.И. Интегралы и ряды. Специальные функции. М.: Наука, 1983.

Письма в ЖТФ, 2017, том 43, вып. 13 